\title{
Erratum to: Cottingham formula and nucleon polarisabilities
}

\author{
J. Gasser ${ }^{1}$, M. Hoferichter ${ }^{1,2,3,4}$, H. Leutwyler ${ }^{1}$, A. Rusetsky ${ }^{5, a}$ \\ ${ }^{1}$ Albert Einstein Center for Fundamental Physics, Institut für theoretische Physik, Universität Bern, Sidlerstrasse 5, 3012 Bern, Switzerland \\ ${ }^{2}$ Institut für Kernphysik, Technische Universität Darmstadt, 64289 Darmstadt, Germany \\ ${ }^{3}$ ExtreMe Matter Institute EMMI, GSI Helmholtzzentrum für Schwerionenforschung GmbH, 64291 Darmstadt, Germany \\ ${ }^{4}$ Institute for Nuclear Theory, University of Washington, Seattle, WA 98195-1550, USA \\ ${ }^{5}$ Helmholtz-Institut für Strahlen- und Kernphysik (Theorie) and Bethe Center for Theoretical Physics, Universität Bonn, Nussallee 14-16, \\ 53115 Bonn, Germany
}

Received: 30 March 2020 / Accepted: 2 April 2020 / Published online: 2 May 2020

(C) The Author(s) 2020

\section{Erratum to: Eur. Phys. J. C https://doi.org/10.1140/epjc/s10052-015-3580-9}

In Ref. [1], the following corrections should be applied: Eq. (10): the last bracket in the third line should be removed. Eq. (27): the factor $v^{2}$ should be replaced by $v^{2}+Q^{2}$.

Eq. (56): $j_{v}(y)$ should be replaced by $j_{\mu}(y)$.

Eq. (57): the factor $m$ in the denominator should be omitted. Eq. (63): $d Q^{2} Q^{2}$ should be replaced by $d Q^{2}$.

Eq. (B.46): the minus sign on the r.h.s. of the second line should be removed.

Open Access This article is licensed under a Creative Commons Attribution 4.0 International License, which permits use, sharing, adaptation, distribution and reproduction in any medium or format, as long as you give appropriate credit to the original author(s) and the source, provide a link to the Creative Commons licence, and indicate if changes were made. The images or other third party material in this article are included in the article's Creative Commons licence, unless indicated otherwise in a credit line to the material. If material is not included in the article's Creative Commons licence and your intended use is not permitted by statutory regulation or exceeds the permitted use, you will need to obtain permission directly from the copyright holder. To view a copy of this licence, visit http://creativecomm ons.org/licenses/by/4.0/.

Funded by SCOAP ${ }^{3}$.

\section{Reference}

1. J. Gasser, M. Hoferichter, H. Leutwyler, A. Rusetsky, Cottingham formula and nucleon polarisabilities. Eur. Phys. J. C 75, 375 (2015). arXiv: 1506.06747

The original article can be found online at https://doi.org/10.1140/ epjc/s10052-015-3580-9.

\footnotetext{
a e-mail: rusetsky@ hiskp.uni-bonn.de (corresponding author)
} 